\title{
AN INTERPRETATION OF THE INDEX OF INERTIA OF THE DISCRIMINANT MATRICES OF A LINEAR ASSOCIATIVE ALGEBRA*
}

\author{
BY \\ R. F. RINEHART
}

1. Introduction. A famous result in the theory of algebraic equations, which was the culmination of researches of Sturm, Sylvester, Hermite, and others, is the so-called Borchardt-Jacobi Theorem, hereinafter referred to as the B. J. Theorem: $\dagger$ Let $f(x)=0$ be a polynomial equation of degree $n$ with real coefficients, and let $s_{i}, i>0$, denote the sum of the ith powers of the roots of $f(x)=0$.

I. The rank of the matrix

$$
T=\left\|\begin{array}{ll}
n & s_{1} \cdots s_{n-1} \\
s_{1} & s_{2} \cdots s_{n} \\
\cdot & \cdot \cdots \cdot \\
s_{n-1} & s_{n} \cdots s_{2 n-2}
\end{array}\right\|
$$

is equal to the number of distinct roots of $f(x)=0$.

II. The signature of $T$ is equal to the number of distinct real roots of $f(x)=0$.

In the theory of linear associative algebras there exists a generalization of part I of this theorem. Let $\mathfrak{A}$ be a linear associative algebra of order $n$ over a field $\Re$ of infinite characteristic, and let $b_{1}, b_{2}, \cdots, b_{n}$ be a basis for $\mathfrak{A}$. Let $c_{i j k},(i, j, k=1, \cdots, n)$, be the constants of multiplication relative to this basis. Then $b_{r} b_{s}=\sum_{t=1}^{n} c_{r s i} b_{i},(r, s=1, \cdots, n)$. The first and second discriminant matrices of $\mathfrak{A}$, relative to this basis, are defined to be, respectively,

$$
\begin{aligned}
& T_{1}(\mathfrak{U})=\left\|t_{1}\left(b_{r} b_{s}\right)\right\|=\left\|\sum_{i=1}^{n} c_{r s i} t_{1}\left(b_{i}\right)\right\|=\left\|\sum_{i, j=1}^{n} c_{r s i} c_{i j j}\right\|, \\
& T_{2}(\mathfrak{U})=\left\|t_{2}\left(b_{r} b_{s}\right)\right\|=\left\|\sum_{i=1}^{n} c_{r s i} t_{2}\left(b_{i}\right)\right\|=\left\|\sum_{i, j=1}^{n} c_{r s i} c_{j i j}\right\|,
\end{aligned}
$$

where $t_{1}\left(b_{i}\right)$ and $t_{2}\left(b_{i}\right)$ are respectively the first and second traces of the element $b_{i}$, that is, the traces of the first and second matrices, $\left\|c_{i s r}\right\|$ and $\left\|c_{r i s}\right\|$,

* Presented to the Society, November 26, 1938; received by the editors March 6, 1939.

$\dagger$ For a complete historical account of this theorem, see the tract Abhandlung ïber die Auflösung der numerischen Gleichungen (Ostwald's Klassiker der exakten Wissenschaften, no. 143), by C. Sturm, edited by A. Loewy, Leipzig, 1904. 
of the element $b_{i}$. It has been shown that $T_{1}(\mathfrak{H})$ and $T_{2}(\mathfrak{H})$ are symmetric,* and that under a transformation of basis of $\mathfrak{A}, b_{i}^{\prime}=\sum_{j=1}^{n} m_{i j} b_{i},(i=1, \cdots, n)$, of matrix $M=\left\|m_{r s}\right\|,\left|m_{r s}\right| \neq 0$, the discriminant matrices are transformed by congruence, ${ }^{*} \dagger$ namely,

$$
T_{1}^{\prime}=M T_{1} M^{T}, \quad T_{2}^{\prime}=M T_{2} M^{T}, \ddagger
$$

so that the ranks (and signatures, if $\Omega$ is an ordered field) of $T_{1}$ and $T_{2}$ are invariant under transformation of basis of $\mathfrak{A}$. The following theorem is well known in the theory of linear algebras:

THEOREM A.§ The nullity of $T_{1}(\mathfrak{A})\left[\right.$ or $\left.T_{2}(\mathfrak{A})\right]$ is equal to the order of the radical of $\mathfrak{A}$.

MacDuffee (cf. M1) has pointed out that the discriminant matrices of the polynomial algebra generated by an element $x$ whose minimum equation is the polynomial equation $f(x)=0$ of degree $n$, relative to the basis $1, x$, $x^{2}, \cdots, x^{n-1}$ become the matrix $T$ of the B. J. Theorem. It has also been noted that, for such an algebra, Theorem A specializes precisely to part I of the B. J. Theorem, $\|$ so that Theorem A is a direct extension of part I of the B. J. Theorem from the case of a polynomial algebra to that of an arbitrary associative algebra.

From this standpoint it is apparent that Theorem A constitutes an incomplete generalization of the B. J. Theorem. An extension of part II of the B. J. Theorem to an arbitrary algebra $\uparrow$ would be desirable. Moreover, when the ground field $\mathfrak{\Omega}$ of the algebra $\mathfrak{A}$ is the real field, the rank and signature of $T_{1}(\mathfrak{A})\left[T_{2}(\mathfrak{A})\right]$ constitute a complete set of invariants of $T_{1}(\mathfrak{A})\left[T_{2}(\mathfrak{A})\right]$ under transformations of basis of $\mathfrak{A}$. Thus, in view of Theorem $A$, if an interpretation of the signature (or any second invariant which is independent of the rank) of $T_{1}(\mathfrak{A})\left[T_{2}(\mathfrak{A})\right]$ is found, then the significance of the discriminant matrices of an algebra over the real field will be, in a sense, fully known.

It is the purpose of this paper to complete the generalization of the B. J. Theorem, and thus exhibit the significance of a complete set of invariants,

* C. C. MacDuffee, The discriminant matrices of a linear associative algebra, Annals of Mathematics, (2), vol. 32 (1931), pp. 60-66; hereinafter referred to as M1.

$\dagger$ C. C. MacDuffee, The discriminant matrix of a semisimple algebra, these Transactions, vol. 33 (1931), pp. 425-432; hereinafter referred to as M2. E. Noether, Mathematische Zeitschrift, vol. 30 (1929), p. 689.

$\ddagger M^{T}$ denotes the transpose of $M$.

§ Cf. L. E. Dickson, Algebren und ihre Zahlentheorie, Zurich, 1927, pp. 108-110.

\| R. F. Rinehart, Bulletin of the American Mathematical Society, vol. 42 (1936), pp. 570-576; hereinafter referred to as $\mathrm{R} 1$.

I Hereafter the term algebra will be understood to denote a linear associative algebra of finite order. 
over the real field, of $T_{1}(\mathfrak{A}) \quad\left[T_{2}(\mathfrak{H})\right]$. The second invariant of $T_{1}(\mathfrak{A}) \quad\left[T_{2}(\mathfrak{A})\right]$ which seems to be most easily interpreted is $\mu$, the number of nonnegative terms in a diagonal canonical form of $T_{1}(\mathfrak{U})\left[T_{2}(\mathfrak{H})\right] *^{*}$ In terms of the order $n$, rank $\rho$, and signature $\sigma$ of $T_{1}(\mathfrak{U})\left[T_{2}(\mathfrak{U})\right], \mu=n-(\rho-\sigma) / 2$. The method of attack on the problem of interpretation is simple in motif but somewhat complicated in the details. In $\S 2$ it is shown that if $\mathfrak{A}$ is simple, $\mu$ is equal to the number in a complete set of primitive idempotents of $\mathfrak{A}$, plus the order of a nilpotent subalgebra of $\mathfrak{A}$ of maximal order. In $\$ 3$ the results of $\$ 2$ are extended to semisimple algebras by the obvious device of applying the classical theorem concerning the decomposition of a semisimple algebra into a direct sum of simple algebras. In $\S \S 4$ and 5 the results of $\$ 3$ are generalized to an arbitrary algebra by again making use of a well known structure theorem to the effect that an arbitrary algebra is the sum of its radical and semisimple algebra. $\nmid$ In $\$ 6$ it is shown that the general theorem of $\$ 5$ specializes to part II of the B. J. Theorem, when the algebra is taken to be a polynomial algebra.

2. The inertia of the discriminant matrix of a simple algebra. $\ddagger$ Let $\mathfrak{D}$ be a division algebra over the real field $\Re$. Then, as is well known, $\mathfrak{D}$ is equivalent to one of (I) the real field $\Re$; (II) the complex field $\mathfrak{S}$; (III) the algebra of real quaternions $\mathfrak{Q}$. If we choose the customary canonical bases

(I) $1: 1^{2}=1$,

(II) $1, i: 1 \cdot i=i \cdot 1=i, i^{2}=-1$,

(III) $1, i, j, k: 1 \cdot i=i \cdot 1=i, 1 \cdot j=j \cdot 1=j, 1 \cdot k=k \cdot 1=k, 1^{2}=1, i^{2}=j^{2}=k^{2}=$ $-1, i j=-j i=k, j k=-k j=i, k i=-i k=j$,

respectively, in cases (I), (II), and (III), the discriminant matrix of $\mathfrak{D}$ assumes the respective forms
(I): $\|1\|$,
(II): $\left\|\begin{array}{rr}2 & 0 \\ 0 & -2\end{array}\right\|$,
(III): $\left\|\begin{array}{rrrr}4 & 0 & 0 & 0 \\ 0 & -4 & 0 & 0 \\ 0 & 0 & -4 & 0 \\ 0 & 0 & 0 & -4\end{array}\right\|$.

* It is shown in $\S 4$ that the signatures (and consequently the invariants $\mu$ ) of $T_{1}(\mathfrak{H})$ and $T_{2}(\mathfrak{H})$ are equal.

$\dagger$ Here difficulty is encountered because, while the interpretation is additive under the operation of "tacking on a radical" to a semisimple algebra, it is not easy to show that $\mu$ possesses the additive property. It seems to the writer that the fundamental Theorem 4.1 should be susceptible of a simpler proof, but such a proof was not found.

$\ddagger$ MacDuffee (M2) has shown that the first and second discriminant matrices of a semisimple algebra over a field of infinite characteristic are equal relative to any given basis. Consequently, for semisimple algebras, the phrase the discriminant matrix is unambiguous. (The terminology infinite characteristic is used in lieu of the customary term characteristic 0 . As has been noted by A. A. Albert (Modern Higher Algebra, University of Chicago Press, 1937), the former nomenclature seems to be more harmonious with the general definition of the characteristic in other cases.) 
In each case the index of inertia $\mu$ of the discriminant matrix is unity, and no clue as to the interpretation of $\mu$ is apparent from these instances. Let us investigate the most general type of simple algebra over $\Re$.

Let $\subseteq$ be a simple algebra over $\Re$. By Wedderburn's well known theorem, $\subseteq$ is equivalent* to a total matric algebra $\mathfrak{M}$ over a division algebra $\mathfrak{D}$. As remarked above $\mathfrak{D}$ must be equivalent to one of $\Re$, $\mathfrak{S}$, or $\mathfrak{Q}$. To interpret the index of inertia of $T(S)$, the following theorem (which was discovered inductively) is of primary importance:

THEOREM 2.1. Let $\subseteq$ be a simple algebra over $\Re$ of order $\delta n^{2}$, where $\delta=1,2$, or 4 according as $\mathfrak{D}$ is $\Re$, $\mathfrak{S}$, or $\mathfrak{Q}$. The order of a nilpotent subalgebra of $\mathfrak{S}$ of maximal order is on $(n-1) / 2$.

We note first that if $n=1, \subseteq$ is a division algebra and hence possesses no nilpotent elements, so that the order of a nilpotent subalgebra of maximal order is zero. Thus Theorem 2.1 is verified when $n=1$.

Now let $n>1$, and let $e_{p q},(p, q=1,2, \cdots, n)$, be the customary basis for the total matric algebra $\mathfrak{M}$; that is, a basis having the multiplication table

$$
e_{p q} e_{l m}=\delta_{q l} e_{p m}, \quad p, q, l, m=1, \cdots, n,
$$

where $\delta_{q l}$ is Kronecker's delta. Let $\mathfrak{R}$ denote the linear form module over $\mathfrak{D}$, a basis for which is $e_{p q},(p=1, \cdots, n-1 ; q=p+1, \cdots, n)$. Then $\&$ is composed of all matrices of the form

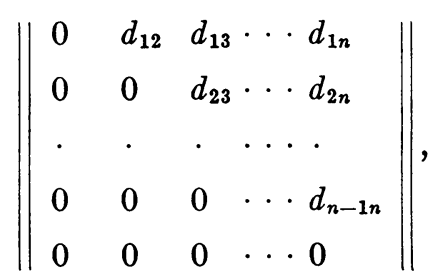

where the $d_{p q}$ are in $\mathfrak{D}$. It is clear that the product of any two elements of $\mathbb{R}$ is again in $\mathfrak{R}$ so that $\mathfrak{R}$ is an algebra. Furthermore, it is apparent that $\mathfrak{R}$ is nilpotent, since the $n$th power of any matrix of the above form is zero. $\dagger$ Hence $\mathfrak{R}$ is a nilpotent subalgebra of $\mathfrak{M}$. Its order is $n(n-1) / 2$. Hence $\subseteq$ has a nilpotent subalgebra of order $\delta n(n-1) / 2$. A basis for this subalgebra is $d_{h} e_{p q}, h=1, \cdots, \delta ; p=1, \cdots, n-1 ; q=p+1, \cdots, n$, where the $d_{h}$ are basis elements of $\mathfrak{D}$.

We wish to show that $\delta n(n-1) / 2$ is the maximal order that a nilpotent

* Two algebras $\mathscr{A}$ and $\mathscr{B}$ will be said to be equivalent, if a simple ring isomorphism exists between the elements of $\mathfrak{A}$ and those of $\mathfrak{B}$.

$\dagger$ To prove these statements one needs only the assumption of the associativity of the elements of the matrices of $\mathbb{R}$. 
subalgebra of $\mathfrak{S}$ may have. For this purpose we need a lemma which we now interrupt the proof of Theorem 2.1 to establish.

LEMMA 1. If a set of $n$ matrices of order $n$ of the type

$$
M_{h}=\sum_{l=1}^{n} d_{h l} e_{h l}, \quad h=1, \cdots, n,
$$

where the $d_{h l}$ are elements of a division algebra $\mathfrak{D}$, is such that every linear combination of them, with coefficients in the ground field $\Omega$ of $\mathfrak{D}$, is nilpotent, then one of the $M_{h}$ is zero.

The proof will be made by mathematical induction on $n$. If $n=1$, $M_{1}=\left(d_{11}\right)$, where $d_{11}$ is in $\mathfrak{D}$. The hypothesis that $M_{1}$ is nilpotent implies that $d_{11}=0$. Thus Lemma 1 holds for $n=1$.

Now assume the lemma to be true for order $n-1$, and consider the case of order $n$. Then

$$
M_{1}=\left\|\begin{array}{cccc}
d_{11} & d_{12} & \cdots & d_{1 n} \\
0 & 0 & \cdots & 0 \\
\cdot & . & \cdots & . \\
0 & 0 & \cdots & 0
\end{array}\right\|, M_{2}=\left\|\begin{array}{cccc}
0 & 0 & \cdots & 0 \\
d_{21} & d_{22} & \cdots & d_{2 n} \\
\cdot & \cdot & \cdots & . \\
0 & 0 & \cdots & 0
\end{array}\right\|, \cdots, M_{n}=\left\|\begin{array}{cccc}
0 & 0 & \cdots & 0 \\
0 & 0 & \cdots & 0 \\
\cdot & \cdot & \cdots & 0 \\
d_{n 1} & d_{n 2} & \cdots & d_{n n}
\end{array}\right\| \text {. }
$$

Consider the matrices of the form

$$
\bar{M}_{1}=c_{2} M_{2}+c_{3} M_{3}+\cdots+c_{n} M_{n}=\left\|\begin{array}{cccc}
0 & 0 & \cdots & 0 \\
c_{2} d_{21} & c_{2} d_{22} & \cdots & c_{2} d_{2 n} \\
\cdot & \cdot & \cdots & \cdot \\
c_{n} d_{n 1} & c_{n} d_{n 2} & \cdots & c_{n} d_{n n}
\end{array}\right\| \text {, }
$$

where the $c_{h}$ are arbitrary elements of $\Omega$. By the hypothesis of the lemma, every such matrix must be nilpotent. This evidently implies that the submatrix of order $n-1$, which is composed of the last $n-1$ rows and columns of $\bar{M}_{1}$, is nilpotent. Since the $c_{h}$ are arbitrary, the assumption of the truth of the lemma for matrices of order $n-1$ implies that one of the rows of this submatrix consists of zeros. Hence one of the matrices $M_{h}$, say $M_{h_{1}}$, is of the form

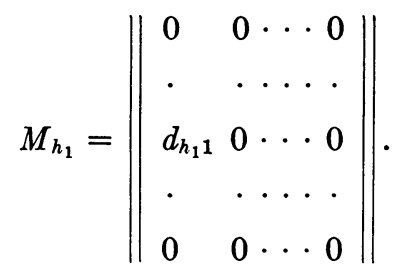

If $d_{h_{1} 1}=0$, the lemma is proved for the case $n=n$. If $d_{h_{1} 1} \neq 0$, consider the 
matrix $\bar{M}_{2}=c_{1} M_{1}+c_{3} M_{3}+\cdots+c_{n} M_{n}$, where the $c_{h}$ are arbitrary numbers of $\Omega$. As before, the hypothesis that $\bar{M}_{2}$ is nilpotent implies that the submatrix of $\bar{M}_{2}$ of order $n-1$, which is composed of rows and columns $1,3,4, \cdots, n$ of $\bar{M}_{2}$, is nilpotent. Again, from the assumption of the induction and from the nature of the $c_{h}$, one of the $M_{h}$, say $M_{h_{2}}$, is of the form

$$
M_{h_{2}}=\left\|\begin{array}{cccc}
0 & 0 & \cdots & 0 \\
\cdot & \cdot & \cdots & . \\
0 & d_{h_{2} 2} & \cdots & 0 \\
\cdot & \cdot & \cdots & 0 \\
0 & 0 & \cdots & 0
\end{array}\right\| \text {. }
$$

Furthermore, since $d_{h_{1} 1} \neq 0$, it follows that $h_{2} \neq h_{1}$. As before, if $d_{h_{2} 2}=0$, the lemma is proved for $n=n$. If $d_{h_{2}} \neq 0$, we proceed as in the previous instances, forming the matrix $\bar{M}_{3}$, and find that one of the $M_{h}$ say $M_{h_{3}}$, with $h_{3} \neq h_{1}, h_{2}$, consists of zero elements with the possible exception of the element in the $h_{3}, 3$ position. In the continuation of this process we must finally arrive at an $M_{h_{s}}$ which is zero. For, if this were not the case, the matrix $M=M_{1}+M_{2}$ $+\cdots+M_{n}$ would have exactly $n$ nonzero elements, no two of which would lie in a common row or column. As in the theory of matrices with commutative elements, such a matrix cannot be nilpotent. Indeed, it is easily seen that any power of $M$ will again be a matrix with at most one nonzero element in each row and column. Each such element is a product of nonzero elements of $\mathfrak{D}$, and since $\mathfrak{D}$ is a division algebra, no such product is zero. Thus $M$ is not nilpotent; but this contradicts the hypothesis of the lemma. Therefore some $M_{h}$ is zero, and the lemma is proved.

We return now to Theorem 2.1. We shall make the proof that $\delta n(n-1) / 2$ is the maximum possible order for a nilpotent subalgebra of $\subseteq$, by mathematical induction on $n$. If $n=1, \subseteq$ has no nilpotent subalgebra, and the formula $\delta n(n-1) / 2$ holds.

Assume the formula holds for a total matric algebra of order $n-1$ over $\mathfrak{D}$ and consider the case $n=n$. Suppose that $\subseteq$ contains a nilpotent subalgebra $\mathfrak{I}^{\prime}$ of order $t>\delta n(n-1) / 2$. We shall show that this assumption leads to a contradiction. Let $l_{1}, l_{2}, \cdots, l_{t}$ be a basis for $\ell^{\prime}$. Since $d_{h} e_{p q},(h=1, \cdots, \delta$; $p, q=1, \cdots, n)$, constitute a basis for $\mathfrak{S}$, each $l_{0}$ is expressible as

$$
l_{g}=\sum_{h, p, q} c_{g h p q} d_{h} e_{p q}, \quad g=1,2, \cdots, t,
$$

where the $c_{g h p q}$ are in $\Re$. Now $t>\delta n(n-1) / 2 \geqq \delta(n-1)$. It is therefore possible to eliminate from the right-hand side of (2.1) all terms involving $e_{p r}, p \neq r$, for a fixed index $r$, by forming a proper linear combination, with real coeffi- 
cients, of the $l_{\sigma},(g=1, \cdots, t)$. Such an element of $\mathfrak{R}^{\prime}$ is of the form

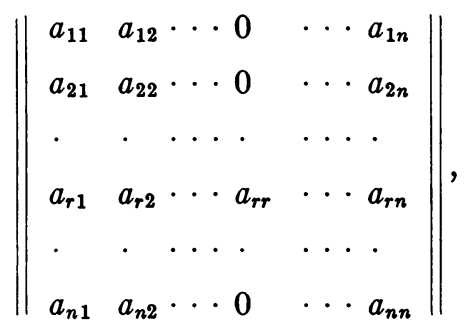

where the $a_{p q}$ are in $\mathfrak{D}$. This matrix is clearly not nilpotent, unless $a_{r r}=0$; therefore, when the $e_{p r}, p \neq r$, for a fixed index $r$, are eliminated from (2.1), $e_{r r}$ is eliminated also.

Since the $l_{o}$ are linearly independent over $\Re$, and since $t>\delta n(n-1) / 2$, it follows from the theory of linear dependence that the number of linearly independent elements of $\mathfrak{R}^{\prime}$, of the form (2.2), for a fixed $r$, is greater than

$$
\frac{1}{2} \delta n(n-1)-\delta(n-1)=\frac{1}{2} \delta(n-1)(n-2) \text {. }
$$

Consider the set of all elements of $\Omega^{\prime}$ of the form (2.2) for a fixed $r$. Every integral rational function of these elements with real coefficients is nilpotent. However, in any such rational integral function, the elements (of the resulting matrix) in the positions $h, m, h \neq r$ and $m \neq r$, are determined completely by the elements of the matrices (2.2) in rows other than the $r$ th and columns other than the $r$ th. In other words the elements of the matrices (2.2) in the $r$ th row or $r$ th column have no effect on rows or columns other than the $r$ th row or column. Therefore the submatrices obtained from (2.2) by deleting row $r$ and column $r$ constitute a nilpotent subalgebra of a total matric algebra of order $(n-1)$ over $\mathfrak{D}$. By the assumption of the induction that the theorem holds for total matric algebras of order $(n-1)$, there can be at most $\delta(n-1)(n-2) / 2$ linearly independent such submatrices of the set (2.2). Since the number of linearly independent matrices of the form (2.2) is greater than $\delta(n-1)(n-2) / 2$, we can, by taking a linear combination of the matrices (2.2), produce a nonzero matrix of the form

$$
\left\|\begin{array}{ccccccc}
0 & \cdots & 0 & 0 & 0 & \cdots & 0 \\
\cdot & \cdots & . & . & . & \cdots & . \\
b_{r 1} & \cdots & b_{r r-1} & 0 & b_{r r+1} & \cdots & b_{r n} \\
0 & \cdots & 0 & 0 & 0 & \cdots & 0 \\
\cdot & \cdots & . & . & . & \cdots & 0 \\
0 & \cdots & 0 & 0 & 0 & \cdots & 0
\end{array}\right\| .
$$

This matrix belongs of course to $\mathfrak{R}^{\prime}$. 
In the above argument $r$ was fixed but arbitrary. Hence a nonzero matrix of $\mathfrak{R}^{\prime}$ of the form (2.3) can be constructed for every $r$ from 1 to $n$. Further, any linear combination of such matrices, with real coefficients, is again in $\mathbb{R}^{\prime}$, and is therefore nilpotent. But this contradicts Lemma 1. Hence the assumption that $\subseteq$, of order $\delta n^{2}$, contains a nilpotent subalgebra of order greater than $\delta n(n-1) / 2$, together with the assumption of the truth of the theorem for smaller values of $n$, leads to a contradiction. This completes the induction proof of Theorem 2.1.

We remark in passing that the nilpotent subalgebra of $\subseteq$ of order $\delta n(n-1) / 2$ is by no means unique. There are many such subalgebras. If a similarity transformation is performed on the elements of one such algebra, one obtains another such algebra, which is equivalent to the first. Whether or not any two nilpotent subalgebras of $\subseteq$ of maximal order are equivalent is a question that the writer has not yet investigated.

We are now in a position to prove

THEOREM 2.2. Let $\mu$ be the index of inertia of the discriminant matrix of a simple algebra $\mathfrak{S}$ over the real field. Let $\epsilon$ be the number in a complete set of primitive idempotents of $\mathfrak{S}$, and let $\chi$ be the order of a nilpotent subalgebra of $\subseteq$ of maximal order. Then $\mu=\epsilon+\chi$.

As previously noted, $\mathfrak{S}$ is either (I) $\Re$, (II) $\mathfrak{S}$, (III) $\mathfrak{Q}$, or (IV) a total matric algebra of order greater than one over one of $\Re$, $\mathbb{E}$, or $\mathfrak{Q}$. In cases (I), (II), and (III) $\mathfrak{S}$ is a division algebra, and hence has no nilpotent elements. Furthermore, it possesses no idempotents other than the principal unit.* Hence $\chi=0$ and $\epsilon=1$. We have seen that if $\subseteq$ is a division algebra, $\mu=1$. Hence Theorem 2.2 holds in cases (I), (II), and (III).

At this point let us recall the following known results:

(a) The discriminant matrix of the direct product of two semisimple algebras $\mathfrak{A}$ and $\mathfrak{B}$ is (for proper choice and ordering of the basis elements) a direct product of the discriminant matrices of $\mathfrak{A}$ and $\mathfrak{B}$ (cf. M2).

(b) The signature of a direct product of two symmetric matrices is equal to the product of the signatures of those matrices. $\dagger$

(c) The signature of the discriminant matrix of a total matric algebra of order $n^{2}$ over the real field is $n$ (cf. M2).

From properties (a), (b), and (c), it follows that, in case (IV), the signature, $\sigma(T(\Im))$, of $T(\widetilde{S})$ is $n, 0$, or $-2 n$, according as $\mathfrak{D}$ is $\Re$, $\mathfrak{C}$, or $\mathfrak{Q}$. For any symmetric matrix, $\mu=(\rho+\sigma) / 2$, where $\rho$ is the rank of the matrix. Since $\subseteq$ is simple, $T(\Im)$ is nonsingular, and hence $\rho(T(\Im))=\delta n^{2}$. Hence according as

* Cf. L. E. Dickson, op. cit., p. 112.

$\dagger$ Cf. C. C. MacDuffee, The Theory of Matrices, Springer, Berlin, 1933, p. 83. 
$\mathfrak{D}$ is $\Re$, $\mathbb{E}$, or $\mathfrak{Q}$, we have, respectively,

(1) $\mu(T(\Im))=\left(n^{2}+n\right) / 2$,

(2) $\mu(T(\Im))=\left(2 n^{2}\right) / 2=n^{2}$,

(3) $\mu(T(\Im))=\left(4 n^{2}-2 n\right) / 2=2 n^{2}-n$.

Now the number in a complete set of primitive idempotents of a total matric algebra over a division algebra is easily seen to be the same as the number of such idempotents of a total matric algebra over a field, namely $n$. By Theorem 2.1 the order $\chi$ of a nilpotent subalgebra of $\subseteq$ of maximal order is $\delta n(n-1) / 2$, where $\delta=1,2$, or 4, respectively, in cases (1), (2), and (3). Hence in the three cases we have

(1) $\chi+\epsilon=n(n-1) / 2+n=n(n+1) / 2=\mu(T(\Im))$,

(2) $\chi+\epsilon=n(n-1)+n=n^{2}=\mu(T(\Im))$,

(3) $\chi+\epsilon=2 n(n-1)+n=2 n^{2}-n=\mu(T(\Im))$, which completes the proof.

It may occur to the reader at this point that Theorem 2.2 can be proved for the more general case where the ground field is any ordered field, for instance the rational field. However, the number of primitive idempotents of an algebra is not invariant under change of ground field, so that Theorem 2.2 is not valid, in general, for an arbitrary ordered field, and in particular, is not valid, in general, for the rational field.

Theorem 2.2 can be put into the alternative form:

THEOREM 2.3. Let $\subseteq$ be a simple algebra over $\Re$, and let $\mathfrak{B}$ be a subalgebra of $\mathfrak{S}$ of minimum order which contains a complete set of primitive idempotents of $\mathfrak{S}$, and which has, as its radical, a nilpotent subalgebra of $\subseteq$ of maximum order. Then the order of $\mathfrak{B}$ is equal to $\mu(T(\Im))$.

To prove this theorem it is sufficient to exhibit a $\mathscr{B}$ whose order is $\mu(T(\Im))$, since no algebra of the type of $\mathfrak{B}$ of the theorem can have an order smaller than $\mu(T(S))$. Such an algebra is that of all matrices of the form

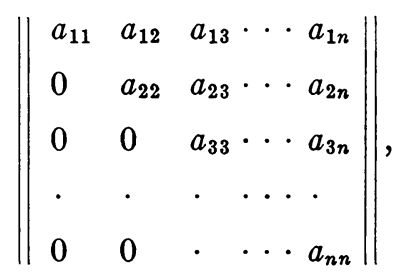

where the $a_{r r}$ are arbitrary real numbers, and the $a_{r s}, r<s$, are arbitrary elements of $\mathfrak{D}$.

3. Extension to semisimple algebras. The method of extension of the results of $\S 2$ to a semisimple algebra is fairly apparent. Let $\mathfrak{A}$ be a semisimple 
algebra over $\Re$. By the well known decomposition theorem, $\mathfrak{A}$ is equivalent to a direct sum of simple algebras $\mathfrak{\Im}_{1}, \mathfrak{\Im}_{2}, \cdots, \mathfrak{\Im}_{\beta}$. It is clear that a nilpotent subalgebra of $\mathfrak{A}$ of maximal order will be a direct sum of such nilpotent subalgebras of the $\mathfrak{S}_{h}$. Further, a complete set of primitive idempotents of $\mathfrak{A}$ will be composed of the complete sets of primitive idempotents of the $\widetilde{S}_{h}$.

On the other hand, for a proper choice of basis of $\mathfrak{A}, T(\mathfrak{U})$ is a direct sum of the discriminant matrices of the $\Im_{h}$ (cf. M2). Moreover, the rank of a direct sum of matrices is equal to the sum of the ranks of the component matrices, and the same is true of the signature when the matrices are symmetric. Hence the index of inertia of $T(\mathfrak{A})$ is equal to the sum of the indices of inertia of the $T\left(\mathfrak{S}_{h}\right)$. This proves

THEOREM 3.1. Let $\mathfrak{A}$ be a semisimple algebra over $\Re$, and let $\epsilon$ be the number in a complete set of primitive idempotents of $\mathfrak{A}$, and $\chi$ the order of a nilpotent subalgebra of $\mathfrak{A}$ of maximal order. Then $\mu(T(\mathfrak{I}))=\chi+\epsilon$.

It is clear that Theorem 2.3 becomes

TheOREm 3.2. Let $\mathfrak{A}$ be a semisimple algebra over $\mathfrak{R}$, and let $\mathfrak{B}$ be a subalgebra of $\mathfrak{A}$ of minimum order, which contains a complete set of primitive idempotents of $\mathfrak{A}$, and which has, as its radical, a nilpotent subalgebra of $\mathfrak{A}$ of maximum order. Then the order of $\mathfrak{B}$ is equal to $\mu(T(\mathfrak{A}))$.

Let $\mathfrak{A}$ be an algebra of order $n$ over a subfield $\Re$ of the real field $\Re$. If the signature of $T_{1}(\mathfrak{A})$ is equal to $n, T_{1}(\mathfrak{U})$ is nonsingular, and therefore $\mathfrak{A}$ is semisimple. Then $T_{1}(\mathfrak{U})=T_{2}(\mathfrak{U})=T(\mathfrak{U})$. Let $\mathfrak{U}^{\prime}$ denote the algebra $\mathfrak{A}$ taken over the real field. Then $\mathfrak{A}^{\prime}$ is equivalent to a direct sum of simple algebras each of which has a discriminant matrix whose signature is equal to its order. From $\$ 2$ the only simple algebra whose order is equal to the signature of its discriminant matrix is the real field itself. Hence $\mathfrak{A}^{\prime}$ is equivalent to a direct sum of algebras of order one, each of which is equivalent to $\Re$. Consequently $\mathfrak{A}^{\prime}$, and therefore $\mathfrak{A}$, is commutative. From the theory of polynomial algebras, every semisimple algebra over a field $\Omega$ of infinite characteristic is equivalent to the polynomial algebra generated by a polynomial with coefficients in $\Omega$ and without repeated factors.* This proves

THEOREM 3.3. Let $\mathfrak{A}$ be an algebra of order $n$ over a subfield $\Re$ of $\Re$. If the signature of $T_{1}(\mathfrak{U})\left[T_{2}(\mathfrak{A})\right]$ is $n$, then $\mathfrak{A}$ is equivalent to a polynomial algebra generated by a polynomial of degree $n$ with coefficients in $\Re$ and without repeated factors, and $\mathfrak{A}$ is therefore commutative.

4. The fundamental theorem for the extension to an arbitrary algebra. Let $\mathfrak{A}$ be an arbitrary non-nilpotent associative algebra over $\Re$. Then $\mathfrak{A}$ is

* Cf. R. F. Rinehart, Commutative algebras which are polynomial algebras, Duke Mathematical Journal, vol. 4 (1938), p. 725; hereinafter referred to as R2. 
the sum of its radical $\mathfrak{Z}$, and a semisimple algebra $\mathfrak{A}$, which is equivalent to the difference algebra $\mathfrak{A} / \mathfrak{Z}$. $\dagger$ If a basis for $\mathfrak{A}$ is chosen to consist of a basis for $\mathfrak{A}^{*}$ together with a basis for $\mathbb{Z}$, the first and second discriminant matrices of $\mathfrak{\Re}$ take the form

$$
T_{1}(\mathfrak{R})=\left\|\begin{array}{ll}
A_{1} & 0 \\
0 & 0
\end{array}\right\|, \quad T_{2}(\mathfrak{A})=\left\|\begin{array}{ll}
A_{2} & 0 \\
0 & 0
\end{array}\right\|,
$$

where $A_{1}$ and $A_{2}$ are nonsingular square matrices, whose order is the order of $\mathfrak{A}^{*}$. The matrix $A_{1}\left[A_{2}\right]$ is $\left\|t_{1}\left(a_{r} a_{s}\right)\right\|\left[\left\|t_{2}\left(a_{r} a_{s}\right)\right\|\right]$, where the $a_{h}$ are basis elements of $\mathfrak{2}^{*}$, and where $t_{1}\left(a_{r} a_{s}\right)\left[t_{2}\left(a_{r} a_{s}\right)\right]$ is the trace of the first [second] matrix of the element $a_{r} a_{s}$ in the representation of $\mathfrak{A}$ by its first [second] matrices (cf. M2 and R1). $\ddagger$

In working with an algebra $\mathbb{S}$ and a subalgebra $\mathfrak{B}$ of $\mathfrak{C}$, the notation $T_{1}(\mathfrak{B})\left[T_{2}(\mathfrak{B})\right]$, relative to a given basis of $\mathfrak{B}$, is ambiguous. For $T_{1}(\mathfrak{B})\left[T_{2}(\mathfrak{B})\right]$ may be formed from the traces of the matrices of the elements of $\mathfrak{B}$ in the representation of $\mathfrak{B}$ by its first [second] matrices, or from the traces of the matrices of the elements of $\mathscr{B}$ in the representation of $\subseteq$ by its first [second] matrices. To avoid this ambiguity we introduce the notation ${ }_{\mathbb{}} T_{1}(\mathfrak{B})\left[{ }_{\S} T_{2}(\mathfrak{B})\right]$, to indicate that $T_{1}(\mathfrak{B})\left[T_{2}(\mathfrak{B})\right]$ is formed from the traces of the matrices in the representation of $\left[5\right.$ by first [second] matrices. For ${ }_{\mathfrak{B}} T_{1}(\mathfrak{B})\left[{ }_{\mathfrak{B}} T_{2}(\mathfrak{B})\right]$ we shall write simply $T_{1}(\mathfrak{B})\left[T_{2}(\mathfrak{B})\right]$, when no confusion is likely to result.

In terms of this notation it is readily seen that the matrices $A_{1}$ and $A_{2}$ of the first paragraph are, respectively, $\mathfrak{\Re}_{1} T_{1}\left(\mathfrak{I}^{*}\right)$ and $\mathfrak{\Re}_{2} T_{2}\left(\mathfrak{R}^{*}\right)$, relative to the basis chosen for $\mathfrak{A}$. The ranks of $\mathfrak{A}_{1} T_{1}\left(\mathfrak{H}^{*}\right), \mathfrak{\Re} T_{2}\left(\mathfrak{A}^{*}\right)$, and $T\left(\mathfrak{A}^{*}\right)$ are equal, for, since $\mathfrak{A}^{*}$ is semisimple, $T\left(\mathfrak{A}^{*}\right)$ is nonsingular. As a first step in the extension of Theorem 3.1 to an arbitrary algebra we shall prove that the signatures of ${ }_{9} T_{1}\left(\mathfrak{A}^{*}\right),{ }_{\mathfrak{A}} T_{2}\left(\mathfrak{H}^{*}\right)$, and $T\left(\mathfrak{A}^{*}\right)$ are likewise equal. For this purpose we need several lemmas, which we shall establish presently.

Let $\subseteq$ be a simple algebra over $\mathfrak{R}$. $\subseteq$ is a total matric algebra $\mathfrak{M}$ over a division algebra $\mathfrak{D}$, which is equivalent to $\Re$, $\mathfrak{C}$, or $\mathfrak{Q}$. Let the canonical basis

$$
d_{h} e_{p q}, \quad h=1, \cdots, \delta ; p, q=1, \cdots, n,
$$

where the $d_{h}$ are a canonical basis for $\mathfrak{D}$, and the $e_{p q}$ a canonical basis for $\mathfrak{M}$, be chosen for $\subseteq$. For this choice of basis all the constants of multiplication are rational. Let $\subseteq^{\prime}$ denote the algebra with the basis (4.1) over the rational field. We shall prove

Lemma 2. A basis for $\mathbb{S}^{\prime}, b_{1}, b_{2}, \cdots, b_{\alpha}, \alpha=\delta n^{2}$, can be so chosen that the minimum equation of each element $b_{h}$ is irreducible in the rational field.

$\dagger$ Cf. L. E. Dickson, ibid., p. 136.

$\ddagger$ Cf. also L. E. Bush, Bulletin of the American Mathematical Society, vol. 38 (1932), pp. 49-51. 
If $\varsigma^{\prime}$ is a division algebra, that is, if $n=1$, then the canonical basis noted at the beginning of $\$ 2$ is a basis of the kind described in the lemma. For, every basis element satisfies one or the other of the equations, $\lambda-1=0, \lambda^{2}+1=0$, each of which is irreducible in the rational field $\mathfrak{F}$.

Suppose now that $n>1$, and suppose that in attempting to choose a basis of the required sort, we have chosen linearly independent elements $b_{1}, b_{2}, \cdots, b_{p}$ each of which satisfies an equation irreducible in $\mathfrak{F}$. Suppose that $p<\alpha$ and that it is impossible to choose another element of $\mathfrak{S}^{\prime}$ which satisfies an equation irreducible in $\mathfrak{F}$ and which is linearly independent of $b_{1}, b_{2}, \cdots, b_{p}$. Let $b_{p+1}, \cdots, b_{\alpha}$ be chosen in any way to fill out a basis for $\mathfrak{S}^{\prime}$. Then the assumption just made implies that every rational linear combination

$$
\sum_{h=1}^{\alpha} c_{h} b_{h}
$$

where at least one of the $c_{h}, h>p$, is different from zero, satisfies a minimum equation which is reducible in $\mathfrak{F}$.

Consider the element $b_{p+1}$ of $\mathfrak{S}^{\prime}$. It is a matrix of order $n$ with elements in $\mathfrak{D}$ not all of which are zero. Let the $r, s$ position be a position in which a nonzero element of the matrix $b_{p+1}$ appears. This element is of the form $a_{0}+a_{1} i+a_{2} j+a_{3} k$, where not all the rational numbers $a_{h}$ are zero. $\dagger$ Since $b_{1}, \cdots, b_{\alpha}$ constitute a basis for $\mathfrak{S}^{\prime}$, we can, by forming linear combinations (4.2) with $c_{p+1} \neq 0$, produce matrices which have some certain one of the elements $1, i, j$, or $k$ in the $r, s$ position, $\ddagger$ and which have any arbitrarily chosen rational linear combinations of $d_{1}, \cdots, d_{\delta}$ in the remaining positions. Now our assumption implies that every matrix of $\varsigma^{\prime}$ which has some certain one of the elements $1, i, j, k$ in the $r, s$ position satisfies a minimum equation which is reducible in $\mathfrak{F}$. We shall show that this leads to a contradiction.

Consider the so-called companion matrix $B$, of order $n$, of the equation $\lambda^{n}-2=0$,

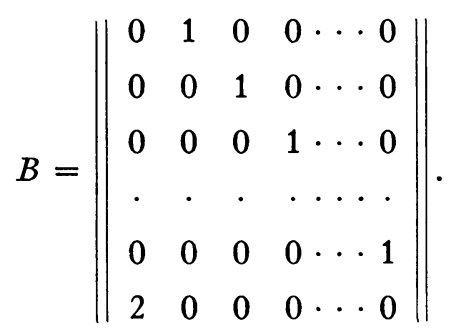

$\dagger$ It is to be understood that if $\mathfrak{D}$ is $\Re, a_{1}=a_{2}=a_{3}=0$, and if $\mathfrak{D}$ is $\mathfrak{S}, a_{2}=a_{8}=0$.

$\ddagger$ For example, if $a_{2} \neq 0$, it is possible to form such matrices with $j$ in the $r, s$ position. 
$\lambda^{n}-2=0$ is the minimum equation of $B$. Furthermore, if $B^{\prime}=P B P^{-1}$ is a matrix similar to $B$, where $P$ is nonsingular with elements in the complex field, then $\lambda^{n}-2=0$ is also the minimum equation of $B^{\prime}$. Now it is fairly evident that a matrix $P$ can be selected so that $P B P^{-1}$ will have a prescribed one of the numbers $1, i, j, k$ in the $r, s$ position. Let $u(1, i, j$, or $k)$ be the element in the $r, s$ position of the matrices constructed in the preceding paragraph. One may verify that, in the several possible cases, the matrices $P$, listed below, will transform $B$ into the matrix $P B P^{-1}$, whose element in the $r, s$ position is $u$.

(1) If $r \neq s-1$ and $s \neq 1, P=I+U_{1}$, where $I$ is the identity matrix, and $U_{1}$ is a matrix with $u$ in the $r,(s-1)$ position and zeros elsewhere.

(2) If $r=s-1$, and $s \neq 1, P$ is a matrix with $u$ in the $r, r$ position, 1's elsewhere on the main diagonal, and zeros in the remaining positions.

(3) If $s=1$ and $r \neq n, P=I+U_{3}$, where $U_{3}$ is a matrix with $u / 2$ in the $r, n$ position and zeros elsewhere.

(4) If $s=1$ and $r=n, P$ is a matrix with $u / 2$ in the $n, n$ position, 1's elsewhere on the main diagonal, and zeros in the remaining positions.

Now in each of the above cases, $P$ has elements which belong to a field which is isomorphic with the complex field, because $u^{2}=1$ or -1 . Hence, in each of the above cases, the matrix $P B P^{-1}$ satisfies the irreducible (in $\mathfrak{F}$ ) equation $\lambda^{n}-2=0$, and has the number $u$ in the $r, s$ position. This contradicts the previous conclusion that a matrix with $u$ in the $r, s$ position should have a minimum equation reducible in $\mathfrak{F}$. Consequently, the initial assumption $p<\alpha$ is untenable, and Lemma 2 is proved.

Let it be remarked that if the basis (4.1) is chosen for $\subseteq$, then the basis $b_{1}, \cdots, b_{\alpha}$ of Lemma 2 can be obtained from (4.1) by a rational transformation of basis.

We return now to the consideration of the arbitrary non-nilpotent algebra $\mathfrak{A}$. Since $\mathfrak{H}^{*}$ is semisimple it is equivalent to a direct sum of simple algebras $\mathfrak{S}_{1}, \mathfrak{S}_{2}, \cdots, \mathfrak{S}_{\beta}$, so that

$$
\mathfrak{A}=\mathfrak{A}^{*}+\mathbb{Z}=\mathfrak{S}_{1}+\mathfrak{\Im}_{2}+\cdots+\mathfrak{\Im}_{\beta}+\mathbb{Z} .
$$

Each $\widetilde{S}_{h}$ has a principal unit $e_{h}$, and $e_{h} e_{l}=\delta_{h l} e_{h}$, where $\delta_{h l}$ is Kronecker's delta. 3 can be separated into a sum of $\beta+1$ linear systems

$$
e_{1} \not, e_{2} \rightrightarrows, \cdots, e_{\beta} \rightrightarrows, Z^{\prime},
$$

where $Z^{\prime}$ consists of all the elements of $\not$, for which $a z=0$ for every element $a$ of $\mathfrak{A}^{*} . \dagger$ The linear systems (4.3) are supplementary in their sum, that is, the intersection of any two of them is zero. For, $e_{h} z_{1}=e_{l} z_{2}, h \neq l$, implies that

† L. E. Dickson, op. cit., pp. 128-130. 
$e_{h}\left(e_{h} z_{1}\right)=e_{h} z_{1}=0$; and $e_{h} z_{1}=z^{\prime}$, where $z^{\prime}$ is in $Z^{\prime}$, implies that $e_{h}\left(e_{h} z_{1}\right)=e_{h} z_{1}=0$. Consequently, a set of bases for the linear systems (4.3) constitutes a basis for 3 .

Now any system $e_{h} Z$ is closed under multiplication on the left by elements of $\mathfrak{A}^{*}$, in particular, by elements of the simple algebra $\mathfrak{S}_{h}$, with which it is associated. For, if $a^{*}$ is any element of $\mathfrak{A}^{*}$, then $a^{*}\left(e_{h} \Re\right)=e_{h}\left(a^{*} Z\right) \subseteq e_{h} \mathfrak{Z}$. If $s_{h}$ is in $\varsigma_{h}$, and $h \neq l$, then $s_{h}\left(e_{l} \Re\right)=0$.

We wish to show that if a rational basis of the type of Lemma 2 is chosen

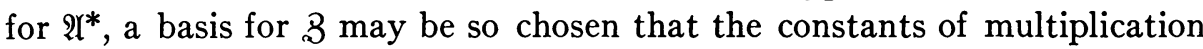
for the product of a basis element of $\mathfrak{A}^{*}$ by a basis element of 3 , in that order, will be rational numbers. To that end we prove

Lemma 3. Let $\subseteq$ be any one of the simple components of $\mathfrak{A}^{*}$, and let e be the principal unit of $\mathfrak{\subseteq}$. Let the canonical basis (4.1) be chosen for $\mathfrak{S}$. Then for this basis of $\mathfrak{\subseteq}$, a basis for $e 3$ can be so chosen that the constants of multiplication for the product of any basis element of $\subseteq$ by any basis element of $e \mathbb{3}$, in that order, will be rational.

If there is an element $z_{1}^{(1)}$ of $\not 3$, for which $e_{11} z_{1}^{(1)} \neq 0$, choose $e_{11} z_{1}^{(1)}$ as one of the basis elements of $e \not 3$. If there is an element $z_{1}^{(2)}$ of 3 such that $e_{11} z_{1}{ }^{(1)}$ and $e_{11} z_{1}{ }^{(2)}$ are left linearly independent over $\mathfrak{D}$, choose $e_{11} z_{1}{ }^{(2)}$ as a second basis element. Continue in this manner, choosing as many further elements $e_{11} z_{1}{ }^{(3)}, \cdots, e_{11} z_{1}{ }^{\left(\nu_{1}\right)}$ as possible which are such that

$$
e_{11}^{(1)} z_{1}^{(1)}, e_{11} z_{1}^{(2)}, \cdots, e_{11} z_{1}^{\left(\nu_{1}\right)}
$$

are left linearly independent over $\mathfrak{D}$. Then any other element of $e \mathfrak{Z}$ of the form $e_{11} z$ is left linearly dependent over $\mathfrak{D}$ on (4.4). When the set (4.4) is thus maximal, or if no element $e_{11} z \neq 0$ exists, select an element $z_{2}^{(1)}$ of 8 which is such that $e_{12} z_{2}{ }^{(1)}$ is left linearly independent over $\mathfrak{D}$ of the elements of (4.4), if such an element $z_{2}{ }^{(1)}$ exists. Take $e_{12} z_{2}{ }^{(1)}$ as a basis element of $e \not 3$. If there is an element $z_{2}{ }^{(2)}$ of 3 which is such that $e_{12} z_{2}{ }^{(2)}$ is left linearly independent over $\mathfrak{D}$ of $e_{12} z_{2}{ }^{(1)}$ and the elements of (4.4), choose $e_{12} z_{2}{ }^{(2)}$ as one of the basis elements of $e \mathbb{3}$. When, in the continuation of this process, the set

$$
e_{12} z_{2}^{(1)}, e_{12} z_{2}^{(2)}, \cdots, e_{12} z_{2}^{\left(\nu_{2}\right)}
$$

is as large as possible, or if no such element $e_{12} z_{2}{ }^{(1)}$ exists, we choose as further basis elements a maximal set

$$
e_{13}^{(1)} z_{3}^{(2)}, e_{13}^{(2)}, \cdots, e_{13} z_{3}^{\left(\nu_{3}\right)},
$$

which, if such elements exist, together with the elements of (4.4) and (4.5) 
are left linearly independent over $\mathfrak{D}$. Continuing in this manner, we finally obtain a set of elements

$$
e_{1 h_{l}} z_{h_{l}}^{\left(m_{l}\right)}, \quad l=1, \cdots, \zeta ; m_{l}=1, \cdots, \nu_{l}, \dagger
$$

where $h_{1}, h_{2}, \cdots, h_{5}$ is some subset of $1,2, \cdots, n$. $\ddagger$ The elements of (4.6) are left linearly independent over $\mathfrak{D}$, and moreover, there is no element of $e \mathfrak{Z}$ of the form $e_{1 h} z$ which is left linearly independent of the elements of (4.6).

Now the elements of the set

$$
e_{p h_{l}} z_{h_{l}}^{\left(m_{l}\right)}
$$$$
p=1,2, \cdots, n ; l=1, \cdots, \zeta ; m_{l}=1, \cdots, \nu_{l},
$$

are left linearly independent over $\mathfrak{D}$. For a relation

$$
\sum_{\substack{p=1, \ldots, n, l=1, \ldots, \zeta, m_{l}=1, \cdots, \nu_{l}}} d_{p h_{l} m_{l}} e_{p h_{l}} z_{h_{l}}^{\left(m_{l}\right)}=0,
$$

where the numbers $d_{p h_{l} m_{l}}$ are in $\mathfrak{D}$, implies, on multiplying on the left by $e_{1_{q}}$,

$$
\sum_{\substack{l=1, \ldots, b_{1} \\ m_{l}=1, \ldots, \nu l}} d_{q h_{l} m_{l}} e_{1 h_{l}} z_{h_{l}}^{\left(m_{l}\right)}=0
$$

for every $q$. But since the elements $e_{1 h_{l}} z_{h_{l}}{ }^{(m) l}$ were chosen to be left linearly independent over $\mathfrak{D},(4.8)$ implies that $d_{q h_{l} m_{l}}=0$ for every $q, h_{l}$, and $m_{l}$. Thus the elements of (4.7) are left linearly independent over $\mathfrak{D}$.

Furthermore, the elements of (4.7) constitute a (left) basis for $e \mathfrak{Z}$ over $\mathfrak{D}$, which may be seen as follows. In the first place, every element of $e \nexists 3$ is the product of $e=e_{11}+e_{22}+\cdots+e_{n n}$ by an element of $\not$, in that order. The existence of an element

$$
e z=e_{11} z+e_{22} z+\cdots+e_{n n} z
$$

of $e \mathfrak{B}$ which is left linearly independent over $\mathfrak{D}$ of the elements (4.7) implies that at least one of the elements $e_{r r} z$ is left linearly independent of (4.7). This implies that $e_{1 r} z$ is also left linearly independent over $\mathfrak{D}$ of $(4.7)$; for, if $e_{1 r} z$ is a left linear combination of the elements of (4.7), then so is $e_{r r} z$, as may be seen by multiplying $e_{1 r} z$ on the left by $e_{r 1}$. But if $e_{1 r} z$ is left linearly independent of (4.7), it is left linearly independent of (4.6). This contradicts the hypothesis that (4.6) is a maximal set.

Consequently, the elements

$\dagger$ It is assumed that $e \mathbb{B} \neq 0$; if $e \mathbb{B}=0$, Lemma 3 is trivially true.

$\ddagger$ If, for instance, there is no element $e_{12} z$ left linearly independent of (4.4) over $\mathfrak{D}$, then 2 will not occur among the $h_{1}, \cdots, h_{\zeta}$. 


$$
\begin{gathered}
d_{q} e_{p h l} z_{h_{l}}^{\left(m_{l)}\right.}, \\
q=1, \cdots, \delta ; p=1, \cdots, n ; l=1, \cdots, \zeta ; m_{l}=1, \cdots, \nu_{l},
\end{gathered}
$$

constitute a basis for $e \mathfrak{Z}$ over $\Re$. For this basis of $e \mathfrak{Z}$ it is clear that the constants of multiplication for the product of a canonical basis element of $\subseteq$ by a basis element of $e ß$, in that order, are rational. In fact these constants of multiplication are 0 's, 1 's, and -1 's.

We remark that if $\subseteq$ is subjected to a rational transformation of basis, from the basis (4.1) to a new basis, and the above basis for $e \geqq 3$ is left unchanged, then the constants of multiplication for the product of a basis element of $\subseteq$ by another basis element of $\mathfrak{S}$, or by a basis element of $e \mathfrak{Z}$, in that order, remain rational. This is true, in particular, for the basis of Lemma 2.

We are now in a position to prove the fundamental theorem on which the extension of the results of $\$ \S 2$ and 3 depends.

THEOREM 4.1. Let $\mathfrak{A}=\mathfrak{A}^{*}+\not$ be an algebra over $\Re$, with the radical $\mathbb{Z}$, and semisimple component $\mathfrak{H}^{*}$. The signatures of $T_{1}(\mathfrak{H}), T_{2}(\mathfrak{H})$, and $\mathfrak{n}^{*} T\left(\mathfrak{H}^{*}\right)$ are equal.

Since $\mathfrak{A}^{*}=\Im_{1} \dot{+} \Im_{2} \dot{+} \cdots \dot{+} \mathfrak{S}_{\beta}$, and $\mathfrak{A}=\mathfrak{A}^{*}+\mathfrak{Z}$, we may choose a basis for $\mathfrak{A}$ by choosing bases for $\mathfrak{S}_{1}, \mathfrak{S}_{2}, \cdots, \mathfrak{S}_{\beta}$ and 3 . Let $e_{1}, e_{2}, \cdots, e_{\beta}$ be the respective principal units of $\mathfrak{S}_{1}, \mathfrak{S}_{2}, \cdots, \mathfrak{S}_{\beta}$. As previously noted, a basis for 3 may be chosen to consist of the bases for such of the systems $e_{1} \rightrightarrows, e_{2} \rightrightarrows, \cdots, e_{\beta} \rightrightarrows, Z^{\prime}$ as are not zero. Let

$$
\begin{aligned}
& s_{1}^{(1)}, \cdots, s_{\alpha_{1}}^{(1)} ; s_{1}^{(2)}, \cdots, s_{\alpha_{2}}^{(2)} ; \cdots ; s_{1}^{(\beta)}, \cdots, s_{\alpha_{\beta}}^{(\beta)} ; \\
& z_{1}^{(1)}, \cdots, z_{\lambda_{1}}^{(1)} ; z_{1}^{(2)}, \cdots, z_{\lambda_{2}}^{(2)} ; \cdots ; z_{1}^{(\beta)}, \cdots, z_{\lambda_{\beta}}^{(\beta)} ; z_{1}, \cdots, z_{\gamma},
\end{aligned}
$$

be a basis for $\mathfrak{A}$, where $s_{1}{ }^{(h)}, \cdots, s_{\alpha_{h}}{ }^{(h)}$ is a basis for $\Im_{h}, z_{1}{ }^{(h)}, \cdots, z_{\lambda_{h}}{ }^{(h)}$ is a basis for $e_{h} \not$, and $z_{1}, \cdots, z_{\gamma}$ is a basis for $\beth^{\prime}$, and where it is to be understood that $z_{1}{ }^{(h)}, \cdots, z_{\lambda_{h}}{ }^{(h)}$ are absent if $e_{h} \not=0$, and similarly for the $z_{p}$, if $\mathfrak{Z}^{\prime}=0$.

Consider any one of the simple algebras $\mathfrak{S}_{h}$. By Lemma 2 the basis elements $s_{1}{ }^{(h)}, \cdots, s_{\alpha_{h}}{ }^{(h)}$ can be taken to be such that each $s_{m}{ }^{(h)}$ satisfies an equation which is irreducible in the rational field $\mathfrak{F}$. Now $s_{m}{ }^{(h)} z=0$ for every element $z$ which is in $e_{1} \not+\cdots+e_{h-1} \not+e_{h+1} \not+\cdots+e_{\beta} \not+Z^{\prime}$. By Lemma 3, if $e_{h} \nsupseteq \neq 0$, the basis $z_{1}{ }^{(h)}, \cdots, z_{\lambda_{h}}{ }^{(h)}$ can be so chosen that the constants of multiplication for a product $s_{m}^{(h)} z_{t}{ }^{(h)}$ are rational.

Consider now the first matrix ${ }_{\mathfrak{q}} R\left(s_{q}^{(h)}\right)$ of any one of the basis elements of $\Xi_{h}$, where $R\left(s_{q}^{(h)}\right)$ denotes the first matrix of $s_{q}^{(h)}$ in the first matric representation of $\mathfrak{A}$ : 


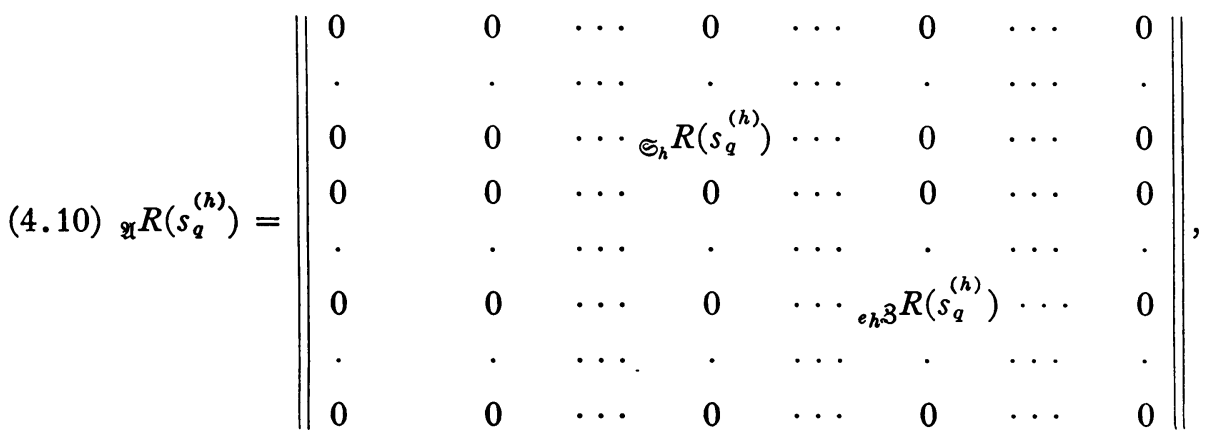

where the 0's stand for blocks of zeros, $\varsigma_{h} R\left(s_{q}^{(h)}\right)$ (occurring in the $h$ th block down and the $h$ th block over) is the first matrix of $s_{q}{ }^{(h)}$ in the first matric representation of $\Im_{h}$, and where $e_{h 3} R\left(s_{q}^{(h)}\right)$ is a matrix of order $\lambda_{h}$, whose elements are the constants of multiplication of products of $s_{q}^{(h)}$ by basis elements of $e_{h} \mathfrak{Z}$. Since $\mathfrak{S}_{h}$ has a principal unit, the matrices $\mathfrak{\varsigma}_{h} R\left(s_{z}^{(h)}\right),\left(q=1,2, \cdots, \alpha_{h}\right)$, are linearly independent. $\dagger$ Hence the matrices ${ }_{\mathfrak{q}} R\left(s_{q}{ }^{(h)}\right)$ are linearly independent, and therefore there is a simple ring isomorphism between the ${ }_{\mathfrak{q}} R\left(s_{q}^{(h)}\right)$ and the $s_{q}{ }^{(h)}$.

The matrix ${ }_{\mathfrak{x}} R\left(e_{h}\right)$ is the matrix (4.10), where ${\varsigma_{h}}_{h} R\left(s_{q}^{(h)}\right)$ and ${ }_{e_{h} 3} R\left(s_{q}^{(h)}\right)$ are identity matrices of orders $\alpha_{h}$ and $\lambda_{h}$, respectively, since $e_{h}$ is a left-hand principal unit for $\mathfrak{S}_{h}$ and $e_{h} 3$. Now each $s_{q}^{(h)},\left(q=1, \cdots, \alpha_{h}\right)$, satisfies an equation

$$
f_{q}(x)=x^{n}+c_{n-1, q} x^{n-1}+\cdots+c_{1 q} x+c_{0 q}=0,
$$

irreducible in $\mathfrak{F}$, when $c_{0 q}$ is replaced by $c_{0 q} e_{h}$. Therefore, $\mathscr{q}_{q} R\left(s_{q}^{(h)}\right)$ also satisfies $f_{q}(x)=0$, if $c_{0 q}$ is replaced by $c_{0 q} \cdot \mathfrak{q} R\left(e_{h}\right)$. Hence, $\varsigma_{h} R\left(s_{q}^{(h)}\right)$ and $e_{h 3} R\left(s_{q}^{(h)}\right)$ also satisfy $f_{q}(x)=0$, when $c_{0 q}$ is replaced respectively by $I_{\alpha_{h}}$ and $I_{\lambda_{h}}$, the identity matrices of orders $\alpha_{h}$ and $\lambda_{h}$. Since the elements of each of $\varsigma_{h} R\left(s_{q}{ }^{(h)}\right)$ and $e_{h} 3 R\left(s_{q}^{(h)}\right)$ are rational, and since $f_{q}(x)$ is irreducible in $\mathfrak{F}, f_{q}(x)$ is the minimum function of each of $\varsigma_{h} R\left(s_{q}^{(h)}\right)$ and $e_{h} 3 R\left(s_{q}{ }^{(h)}\right)$, because the minimum function of a matrix divides any polynomial which vanishes for that matrix. The characteristic function of $\Phi_{h} R\left(s_{q}^{(h)}\right)$ is therefore a power $\left[f_{q}(x)\right]^{\phi}$ of its minimum function $f_{q}(x)$, and hence the trace of $\varsigma_{h} R\left(s_{q}^{(h)}\right)$ is equal to $\phi c_{n-1, q}$. Likewise the characteristic function of $e_{h} 3 R\left(s_{q}{ }^{(h)}\right)$ is a power $\left[f_{q}(x)\right]^{\psi}$ of $f_{q}(x)$, and the trace of $e_{h 3} R\left(s_{q}^{(h)}\right)$ is $\psi c_{n-1, q}$. Now the first trace of $s_{q}^{(h)}$ relative to $\mathfrak{A}, t_{2} t_{1}\left(s_{q}^{(h)}\right)$, is the trace of $\Re_{R} R\left(s_{q}{ }^{(h)}\right)$, which is equal to the sum of the traces of $\varsigma_{h} R\left(s_{q}{ }^{(h)}\right)$ and $e_{h 3} R\left(s_{q}{ }^{(h)}\right)$. Hence

† Cf. L. E. Dickson, op. cit., p. 34. 


$$
\begin{aligned}
\mathfrak{\Re}^{t} t_{1}\left(s_{q}^{(h)}\right) & =t\left({ }_{\mathfrak{Q}} R\left(s_{q}^{(h)}\right)\right)=(\phi+\psi) c_{n-1, q} \\
& =\left(1+\frac{\psi}{\phi}\right) \phi c_{n-1, q}=\left(1+\frac{\lambda_{h}}{\alpha_{h}}\right) \cdot t\left(_{\mathfrak{P}^{*}} R\left(s_{q}^{(h)}\right)\right) .
\end{aligned}
$$

We have arrived at (4.11) on the assumption that $e_{h} \rightrightarrows \neq 0$. However, if $e_{h} 3=0$, then ${ }_{e_{h} 3} R\left(s_{q}^{(h)}\right)$ does not appear in the matrix (4.10). Further, $\lambda_{h}=0$. From this it is apparent that (4.11) also holds if $e_{h} B=0$.

Now $t\left(\mathfrak{q}^{*} R\left(s_{q}^{(h)}\right)\right)=\mathfrak{q}^{*} t_{1}\left(s_{q}^{(h)}\right)$, the first trace of $s_{q}^{(h)}$ relative to $\mathfrak{A}^{*}$. It is known that $t_{1}(a)=t_{2}(a)$, for every element $a$ of a semisimple algebra (cf. R1). Hence we may write (4.11) as

$$
{ }_{\mathscr{q}} t_{1}\left(s_{q}^{(h)}\right)=\theta_{h}\left\lfloor\mathfrak{q}^{*} t\left(s_{q}^{(h)}\right)\right],
$$

where $\theta_{h}=1+\lambda_{h} / \alpha_{h}>0$. Note that $\theta_{h}$ is the same for every element $s_{q}^{(h)}$ of $\Im_{h}$.

Since every element of $\Im_{h}$ is a linear combination of $s_{1}{ }^{(h)}, s_{2}{ }^{(h)}, \cdots, s_{\alpha_{h}}{ }^{(h)}$ with coefficients in $\Re$, and since the trace of a linear combination of elements is equal to the same linear combination of the traces of those elements, it follows from (4.12) that

$$
{ }_{\mathfrak{A}} T_{1}\left(\Im_{h}\right)=\theta_{h}\left[\Im_{h} T\left(\Im_{h}\right)\right] .
$$

Since $\mathfrak{S}_{h}$ was chosen arbitrarily, (4.13) holds for every $h$. Of course $\theta_{h}$ may change with $h$. Hence we may write

$$
T_{1}(\mathfrak{A})=\left\|\begin{array}{cccccc}
\theta_{1} \cdot \Im_{1} T\left(\Im_{1}\right) & 0 & \cdots & 0 & \cdots & 0 \\
0 & \theta_{2} \cdot \Xi_{2} T\left(\Im_{2}\right) & \cdots & 0 & \cdots & 0 \\
\cdot & \cdot & \cdots & . & \cdots & \cdot \\
0 & 0 & \cdots & \theta_{\beta} \cdot \Im_{\beta} T\left(\Im_{\beta}\right) & \cdots & 0 \\
\cdot & \cdot & \cdots & \cdot & \cdots & \cdot \\
0 & 0 & \ldots & 0 & \cdots & 0
\end{array}\right\| .
$$

The signature, $\sigma\left(T_{1}(\mathfrak{H})\right)$, of $T_{1}(\mathfrak{H})$ is the sum of the signatures of the matrices $\theta_{h}\left[\Im_{h} T\left(\Im_{h}\right)\right]$. Since $\theta_{h}>0$, the signature of $\theta_{h}\left[\varsigma_{h} T\left(\Im_{h}\right)\right]$ is the same as the signature of $\Im_{h} T\left(\Im_{h}\right)$. But, since $\mathfrak{A}^{*}$ is the direct sum of the $\Im_{h}$, the sum of the signatures of the $\mathfrak{S}_{h} T\left(\mathfrak{S}_{h}\right)$ is exactly the signature of $\mathfrak{\Re}^{*} T\left(\mathfrak{H}^{*}\right)$. This proves Theorem 4.1 for the signatures of $T_{1}(\mathfrak{A})$ and $\mathfrak{x}^{*} T\left(\mathfrak{A}^{*}\right)$ for a particular basis of I.

Under a transformation of basis of $\mathfrak{A}$ of matrix $C, T_{1}(\mathfrak{A})$ is transformed into $C T_{1}(\mathfrak{H}) C^{T}$, and $\sigma\left(T_{1}(\mathfrak{H})\right)$ is invariant. Now the semisimple algebra $\mathfrak{I}^{*}$ of $\mathfrak{A}$ is not unique, but any two such components are equivalent. Since the discriminant matrices depend only on the constants of multiplication, the 
fact that $\mathfrak{A}^{*}$ and $\mathfrak{A}^{* \prime}$ are equivalent is sufficient to insure the equality of their discriminant matrices for isomorphic bases. Since, further, a transformation of basis of $\mathfrak{A}^{*}$ does not change the signature of $\mathfrak{x}^{*} T\left(\mathfrak{X}^{*}\right)$, it follows that if $\sigma\left(T_{1}(\mathfrak{A})\right)=\sigma\left(T\left(\mathfrak{A}^{*}\right)\right)$ for one choice of basis of $\mathfrak{A}$, the like is true for all bases.

Now it should be evident that if we should make a right-hand decomposition of $\angle$ into the $\beta+1$ linear systems

$$
\not e_{1}, \not e_{2}, \cdots, Z e_{\beta}, Z^{\prime \prime} \text {, }
$$

the analogue of Lemma 3 can be stated and proved in a "right-hand" way. Then the above proof can be carried out in a precisely analogous manner for the equality of the signatures of $T_{2}(\mathfrak{X})$ and $\mathfrak{\Re}^{*} T\left(\mathfrak{H}^{*}\right)$, by use of the second matrices $\Psi_{2} S\left(s_{q}{ }^{(h)}\right)$ of the elements of $\Im_{h}$. The constants $\theta_{h}{ }^{\prime}$ will of course not be necessarily the same as the corresponding $\theta_{h}$. This completes the proof of Theorem 4.1.

We pause briefly to note two corollaries to the proof of Theorem 4.1, which have no direct bearing on the problem of this paper, but which are of interest in their own right. It has been shown that the first and second discriminant matrices of an algebra, relative to a given basis, are not, in general, equal (cf. R1). However, from Theorem A of $\$ 1$ they have the same rank, and by Theorem 4.1 they have the same signature. Moreover,

COROLlaRy 4.11. Let $\mathfrak{B}$ be a primary algebra over $\Re$, with the radical $\mathfrak{Z}$. Then $T_{1}(\mathfrak{B})$ is equal to a scalar times $T_{2}(\mathfrak{B})$.

Since $\mathfrak{B}$ is primary, it is equivalent to the sum of its radical $\mathbb{Z}$ and a simple algebra $\mathbf{5}$. For the particular basis $B$ used in the proof of Theorem 4.1, we have from (4.14)

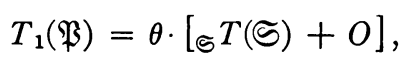

where $\theta$ is a nonzero scalar matrix and $O$ is a zero matrix of order $p$, the order of 3 . Likewise, for another similarly chosen basis $B^{\prime}$ for $\mathfrak{P}$, we would have

$$
T_{2}^{\prime}(\mathfrak{B})=\theta^{\prime} \cdot\left[{ }_{\Im} T(\Im)+O\right],
$$

where $\theta^{\prime}$ is a nonzero scalar matrix. Now the bases $B$ and $B^{\prime}$ for which (4.14) and (4.15) hold, differ only in the choice of basis for the radical 3 . It is therefore possible to make a transformation of basis of $\mathfrak{P}$ from $B$ to $B^{\prime}$, by a transformation whose matrix $Q$ is of the form

$$
Q=I_{\alpha}+M,
$$

where $I_{\alpha}$ is the identity matrix of order $\alpha, \alpha$ being the order of $\subseteq$. Hence, relative to the basis $B^{\prime}, T_{1}(\mathfrak{B})$ becomes 


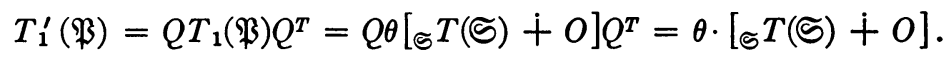

Hence, relative to the basis $B^{\prime}, T_{1}^{\prime}(\mathfrak{B})=\left(\theta / \theta^{\prime}\right) T_{2}^{\prime}(\mathfrak{B})$. Since $T_{1}(\mathfrak{B})$ and $T_{2}(\mathfrak{B})$ are transformed cogrediently under transformations of basis of $\mathfrak{B}$, it follows that $T_{1}(\mathfrak{B})=\left(\theta / \theta^{\prime}\right) T_{2}(\mathfrak{B})$ for every basis of $\mathfrak{B}$.

COROLLARY 4.12. Let $\mathfrak{A}=\mathfrak{A}^{*}+\mathbb{Z}$ be an algebra over $\mathfrak{R}$, with radical $\mathbb{B}$ and semisimple component $\mathfrak{R}^{*}$. It is possible to choose a basis for $\mathfrak{A}$ such that $T_{1}(\mathfrak{A})$, $T_{2}(\mathfrak{A})$, and $\left.\mathfrak{\Re}^{*} \mathfrak{H}^{*}\right)$ simultaneously assume a diagonal form.

A basis for a semisimple algebra for which the discriminant matrix assumes a diagonal form has been called by MacDuffee (M2). a normal basis. Let $\mathfrak{A}=\Im_{1} \dot{+} \Im_{2} \dot{+} \cdots+\mathfrak{\Im}_{\beta}+\mathfrak{Z}$, where the $\Im_{h}$ are simple algebras. Let a basis for $\mathfrak{A}$ be chosen to consist of normal bases for the $\widetilde{S}_{h}$ and any basis for $\mathfrak{Z}$. From the proof of Theorem 4.1, or from Corollary 4.11 we have

$$
{ }_{2} T_{1}\left(\Im_{h}\right)=\theta_{h}\left[\Im_{h} T\left(\Im_{h}\right)\right], \quad{ }_{\mathfrak{q}} T_{2}\left(\Im_{h}\right)=\theta_{h}^{\prime}\left[\Im_{h} T\left(\Im_{h}\right)\right], \quad h=1, \cdots, \beta,
$$

so that for the present basis choice, ${ }_{\mathfrak{A}} T_{1}\left(\Im_{h}\right)$ and ${ }_{\mathfrak{x}} T_{2}\left(\Im_{h}\right)$ are diagonal matrices because $\Im_{h} T\left(\Im_{h}\right)$ is such. But $T_{1}(\mathfrak{H})$ is a direct sum of the $\mathfrak{a} T_{1}\left(\Im_{h}\right)$ and a zero matrix of order equal to the order of 3 . Hence $T_{1}(\mathfrak{A})$ is a diagonal matrix. Similarly, $T_{2}(\mathfrak{A})$, relative to this basis, is a diagonal matrix.

5. Extension to an arbitrary algebra. With the aid of Theorem 4.1 the extension of Theorems 3.1 and 3.2 is readily made. Let $\mathfrak{A}=\mathfrak{Q}^{*}+\mathfrak{Z}$ be an arbitrary algebra over the real field. First, let $\mathfrak{A}$ be non-nilpotent, that is, assume $\mathfrak{A}^{*} \neq 0$. A nilpotent subalgebra $\mathfrak{\&}$ of $\mathfrak{A}$ of maximal order will clearly be the sum of the radical 3 and a nilpotent subalgebra $\mathfrak{\Omega}^{*}$ of $\mathfrak{2}^{*}$ of maximal order. A complete set of primitive idempotents of $\mathfrak{H}^{*}$ also constitutes a complete set of $\mathfrak{A}$. Let $\mu$ denote the number of nonnegative terms in a diagonal form of $T_{1}(\mathfrak{A})$ [or $T_{2}(\mathfrak{A})$ ]. That is, $\mu=n+(\sigma-\rho) / 2$, where $n$ is the order, $\sigma$ the signature, $\rho$ the rank of $T_{1}(\mathfrak{A})$ [or $T_{2}(\mathfrak{A})$ ]. Also $\mu=\mu^{*}+\omega$, where $\mu^{*}$ is the index of inertia of $\mathfrak{x} T_{1}\left(\mathfrak{H}^{*}\right)$ [or $\mathfrak{x} T_{2}\left(\mathfrak{H}^{*}\right)$ ], and where $\omega$ is the common nullity of $T_{1}(\mathfrak{H})$ and $T_{2}(\mathfrak{A})$. From Theorem 4.1 it follows that the indices of inertia of $\mathfrak{n} T_{1}\left(\mathfrak{A}^{*}\right)$, $\mathfrak{x}_{2}\left(\mathfrak{H}^{*}\right)$, and $\mathfrak{\Re}^{*} T\left(\mathfrak{R}^{*}\right)$ are equal. From this and from Theorem $3.1 \mu^{*}=\epsilon+\chi^{*}$, where $\epsilon$ is the number in a complete set of primitive idempotents of $\mathfrak{A}^{*}$ [or $\mathfrak{A}$ ], and $\chi^{*}$ is the order of $\mathfrak{R}^{*}$. Therefore, $\mu=\epsilon+\chi$ where $\chi$ is the order of $\mathbb{R}$.

If $\mathfrak{A}$ is nilpotent, then $\epsilon=0, T_{1}(\mathfrak{U})=T_{2}(\mathfrak{A})=0, \mu=n$, and the relation $\mu=\epsilon+\chi$ is trivially true.

This completes the proof of the general

THEOREM 5.1. Let $\mathfrak{A}$ be an arbitrary algebra of order $n$ over $\Re$. Let $\mu$ $(\mu=n+(\sigma-\rho) / 2$, where $\rho$ and $\sigma$ are, respectively, the rank and signature of $\left.T_{1}(\mathfrak{H})\left[T_{2}(\mathfrak{A})\right]\right)$, be the number of nonnegative terms in the diagonal of a diagonal 
form of $T_{1}(\mathfrak{A})\left[T_{2}(\mathfrak{A})\right]$. Then $\mu$ is equal to the order of a nilpotent subalgebra of $\mathfrak{A}$ of maximal order, plus the number in a complete set of primitive idempotents of $\mathfrak{A}$.

Also, from Theorem 3.2 follows

Theorem 5.2. Let $\mathfrak{A}$ and $\mu$ have the same significance as in Theorem 5.1. Then $\mu$ is equal to the order of a subalgebra of $\mathfrak{A}$ of minimum order which contains a complete set of primitive idempotents of $\mathfrak{A}$, and which has, as its radical, a nilpotent subalgebra of $\mathfrak{A}$ of maximal order.

It may be remarked that a nilpotent subalgebra of $\mathfrak{A}$ of maximal order is also obviously maximal in the sense of the calculus of complexes.

6. Specialization to the Borchardt-Jacobi Theorem. To demonstate how Theorem 5.1 (or 5.2) is a generalization of part II of the B. J. Theorem, let us specialize $\mathfrak{A}$ to a polynomial algebra. Let $p(x)=0$ be a polynomial equation of degree $n$ with real coefficients and with leading coefficient unity. Let $\mathfrak{X}$ be the polynomial algebra over $\Re$ generated by $p(x)$. Over $\Re, p(x)$ can be decomposed into powers of distinct irreducible factors thus:

$$
p(x)=\prod\left(x-a_{i}\right)^{h_{i}}\left(x^{2}+b_{j} x+c_{j}\right)^{k_{i}}, \quad i=1, \cdots, r ; j=1, \cdots, s,
$$

where the $a_{i}, b_{j}$, and $c_{j}$ are in $\Re$. It is known that $\mathfrak{X}$ is equivalent to a direct sum of the $r+s$ polynomial algebras generated by the $\left(x-a_{i}\right)^{h_{i}}$ and the $\left(x^{2}+b_{j} x+c_{j}\right)^{k_{i}}$ (cf. R2). Since $\mathfrak{X}$ is commutative and has a principal unit, the number of primitive idempotents of $\mathfrak{X}$ is equal to the number of primary component algebras in the direct sum decomposition of $\mathfrak{X}$. $†$ Hence $\epsilon=r+s$. Again, because $\mathfrak{X}$ is commutative, the nilpotent subalgebra of $\mathfrak{X}$ of maximal order coincides with the radical of $\mathfrak{X}$, both consisting of all the nilpotent elements of $\mathfrak{X}$. Now the order of the radical of $\mathfrak{X}$ is

$$
\chi=\sum_{i=1}^{r}\left(h_{i}-1\right)+2 \sum_{j=1}^{s}\left(k_{j}-1\right)=n-(r+2 s) .
$$

Hence the rank $\rho$ of the discriminant matrix of $\mathfrak{X}$ is $r+2 s$.

By Theorem 5.1 the signature of $T(\mathfrak{X})$ must be equal to $2(\mu-n)+\rho$ $=2(\chi+\epsilon-n)+\rho$. But $2(\chi+\epsilon-n)+\rho=2[r+s+n-(r+2 s)-n]+r+2 s=r$. Hence the signature of $T(\mathfrak{X})$ is equal to $r$, the number of distinct real roots of $p(x)=0$. This result is part II of the B. J. Theorem.

† See, for instance, G. Scorza, Sulle algebre riducibili, Rendiconti del Seminario Matematico delle Università di Roma, (4), vol. 1 (1937), pp. 188-189.

Case School of Applied Sctence,

Cleveland, Omo 